\title{
EXISTENCE AND EXPONENTIAL STABILITY OF POSITIVE PERIODIC SOLUTIONS FOR SECOND-ORDER DYNAMIC EQUATIONS
}

\author{
Faycal Bouchelaghem
}

High School of Management Sciences Annaba, Annaba 23000, Algeria

fyb1500@gmail.com

\author{
Abdelouaheb Ardjouni \\ Department of Mathematics and Informatics, Souk Ahras University, \\ P.O. Box 1553, Souk Ahras, 41000, Algeria \\ abd_ardjouni@yahoo.fr
}

\author{
Ahcene Djoudi \\ Department of Mathematics, Annaba University, \\ P.O. Box 12, Annaba 23000, Algeria \\ adjoudi@yahoo.com
}

\begin{abstract}
In this article, we establish the existence of positive periodic solutions for second-order dynamic equations on time scales. The main method used here is the Schauder fixed point theorem. The exponential stability of positive periodic solutions is also studied. The results obtained here extend some results in the literature. An example is also given to illustrate this work.

Keywords: Positive periodic solutions, Exponential stability, Schauder fixed point theorem, Dynamic equa-
\end{abstract} tions, Time scales.

\section{Introduction}

Time scales theory was initiated by Stefan Hilger in 1988 as a means of unifying theories from discrete analysis and continuous analysis. Difference equations are defined on discrete sets while differential equations are defined on an interval of the set of real numbers. However, dynamic equations on time scales are very important in the physical applications because they are either difference equations, differential equations or a combination of both. This means that dynamic equations are defined on discrete, connected or combination of both types of sets. Hence, the theory of time scales provides an extension of difference analysis and differential analysis, see $[6,7,15,17]$ and the references therein.

Delay dynamic equations arise in many applications of different fields of science and engineering. For example, these equations appear in applied sciences, physics, chemistry, biology, medicine, etc. In particular, qualitative analysis such as positivity, periodicity and stability of solutions of dynamic equations on time scales has received the attention of many authors, see [1-17] and the references therein.

Let $\mathbb{T}$ be a periodic time scale such that $t_{0} \in \mathbb{T}$. In this paper, we are interested in the positivity, periodicity and exponential stability of solutions of second-order dynamic equations. Inspired and 
motivated by the references in this paper, we consider the following second-order dynamic equation

$$
x^{\triangle \Delta}(t)+a\left(x^{\sigma}\right)^{\Delta}(t)+q(t) x^{\beta}(t)-r(t) x^{\alpha}(t)=0, \quad t \geq t_{0},
$$

with $x^{\Delta}\left(t_{0}\right)+a x^{\sigma}\left(t_{0}\right)=0$ and $x\left(t_{0}\right)=1$. Throughout this paper we assume that $a \geq 0$, $q, r \in C_{r d}\left(\left[t_{0}, \infty\right) \cap \mathbb{T}, \mathbb{R}\right), \alpha, \beta \in(0, \infty)$. To prove the positivity and periodicity of solutions of (1.1), we convert (1.1) into an equivalent integral equation and then employ the Schauder fixed point theorem. The sufficient conditions for the exponential stability of positive solutions are also considered. In the special case $\mathbb{T}=\mathbb{R}$, Dorociakova, Michalkova, Olach and Saga in [13] show the existence and the exponential stability of positive solutions of (1.1). Then, the results presented in this paper extend the main results in [13].

The rest of this work is organized as follows. In Section 2, we present some basic concepts concerning the calculus on time scales that will be used to show our main results. We give some properties of the exponential function on a time scale as well as the Schauder fixed point theorem. We refer the reader to the monograph [18] for more details on the Schauder theorem. In Section 3, we prove our main results for the existence of positive periodic solutions by using the Schauder theorem, and we give an example to illustrate our existence results. In Section 4, we study the exponential stability of a positive periodic solution of (1.1). In Section 5, we establish new sufficient conditions for the existence and the exponential stability for a pipe-tank flow configuration.

\section{Preliminaries}

The theory of dynamic equations is a fairly new branch in mathematics (see [1-10, 14-17]). Dynamic equations extend and unify the difference and differential equations. We assume that most readers are familiar with the basic concepts of the dynamic equations on time scales and for more details we refer to the books $[6,7,17]$.

Definition 1 [6]. A time scale $T$ is an arbitrary nonempty closed subset of $\mathbb{R}$.

The definition of periodic time scales was introduced by Kaufmann and Raffoul [16]. The following two definitions are found in [16].

Definition 2. A time scale $\mathbb{T}$ is said to be periodic provided there exists a $T>0$ such that if $t \in \mathbb{T}$ then $t \pm T \in \mathbb{T}$. For $\mathbb{T} \neq \mathbb{R}$, the period of the time scale is the smallest positive $T$.

Example 1 [16]. The following time scales are periodic.

1. $\mathbb{T}=\bigcup_{i=-\infty}^{\infty}[2(i-1) h, 2 i h], h>0$ has period $T=2 h$.

2. $\mathbb{T}=h \mathbb{Z}$ has period $T=h$.

3. $\mathbb{T}=\mathbb{R}$.

4. $\mathbb{T}=\left\{t=k-q^{m}: k \in \mathbb{Z}, m \in \mathbb{N}_{0}\right\}$ where $0<q<1$ and $\mathbb{N}_{0}$ is the natural numbers with zero, has period $T=1$.

Remark 1 [16]. All periodic time scales are unbounded above and below.

Definition 3. Let $\mathbb{T} \neq \mathbb{R}$ be a periodic time scale with period $T$. The function $f: \mathbb{T} \rightarrow \mathbb{R}$ is said to be periodic with period $\omega$ provided there exists a natural number $n$ such that $\omega=n T$, $f(t \pm \omega)=f(t)$ for all $t \in \mathbb{T}$ and $\omega$ is the smallest number such that $f(t \pm \omega)=f(t)$. If $\mathbb{T}=\mathbb{R}$, $f$ is said to be periodic with period $\omega>0$ provided $\omega$ is the smallest positive number such that $f(t \pm \omega)=f(t)$ for all $t \in \mathbb{T}$. 
Definition 4 [6]. Let $\mathbb{T}$ be a time scale. The forward jump operator $\sigma: \mathbb{T} \rightarrow \mathbb{T}$ is defined by

$$
\sigma(t)=\inf \{s \in \mathbb{T}: s>t\} \quad \text { for all } t \in \mathbb{T},
$$

while the graininess function $\mu: \mathbb{T} \rightarrow[0, \infty)$ is defined by

$$
\mu(t)=\sigma(t)-t \quad \text { for all } \quad t \in \mathbb{T} \text {. }
$$

Remark 2 [16]. Let $\mathbb{T}$ be a periodic time scale with period $T$. Then, the forward jump operator $\sigma$ satisfies $\sigma(t \pm n T)=\sigma(t) \pm n T$. Hence, $\mu(t \pm n T)=\sigma(t \pm n T)-(t \pm n T)=\sigma(t)-t=\mu(t)$. So, $\mu$ is a periodic function with period $T$.

Definition 5 [6]. We say that the function $f: \mathbb{T} \rightarrow \mathbb{R}$ is regulated if its right-sided limits exist at all right-dense points in $\mathbb{T}$ and its left-sided limits exist at all left-dense points in $\mathbb{T}$.

Definition 6 [6]. We say that the function $f: \mathbb{T} \rightarrow \mathbb{R}$ is rd-continuous if it is continuous at every right-dense point $t \in \mathbb{T}$ and its left-sided limits exist, and is finite at every left-dense point $t \in \mathbb{T}$. We denote the set of rd-continuous functions $f: \mathbb{T} \rightarrow \mathbb{R}$ by

$$
C_{r d}=C_{r d}(\mathbb{T})=C_{r d}(\mathbb{T}, \mathbb{R})
$$

We denote the set of differentiable functions $f: \mathbb{T} \rightarrow \mathbb{R}$ and whose derivative is rd-continuous by

$$
C_{r d}^{1}=C_{r d}^{1}(\mathbb{T})=C_{r d}^{1}(\mathbb{T}, \mathbb{R}) .
$$

Definition 7 [6]. The delta derivative $f^{\Delta}(t)$ of a function $f: \mathbb{T} \rightarrow \mathbb{R}$ at a point $t \in \mathbb{T}^{k}=\mathbb{T} \backslash\{\sup \mathbb{T}\}$ exists provided that for any given $\varepsilon>0$, there exists a neighborhood $U$ of $t$ such that

$$
\left|(f(\sigma(t))-f(s))-f^{\Delta}(t)(\sigma(t)-s)\right|<\varepsilon|\sigma(t)-s| \quad \text { for all } \quad s \in U
$$

We say that the function $f^{\Delta}: \mathbb{T}^{k} \rightarrow \mathbb{R}$ is the delta derivative of $f$ on $\mathbb{T}^{k}$.

Definition 8 [6]. A function $p: \mathbb{T} \rightarrow \mathbb{R}$ is said to be regressive if $1+\mu(t) p(t) \neq 0$ for all $t \in \mathbb{T}$. We denote the set of all rd-continuous and regressive functions $p: \mathbb{T} \rightarrow \mathbb{R}$ by $\mathcal{R}=\mathcal{R}(\mathbb{T}, \mathbb{R}) . W e$ define the set $\mathcal{R}^{+}$of all rd-continuous and positively regressive functions by

$$
\mathcal{R}^{+}=\mathcal{R}^{+}(\mathbb{T}, \mathbb{R})=\{p \in \mathcal{R}: 1+\mu(t) p(t)>0, \forall t \in \mathbb{T}\}
$$

Theorem 1 [6]. Suppose $f: \mathbb{T} \rightarrow \mathbb{R}$ is a regulated function. Then there exists a function $F$ which is pre-differentiable with region of differentiation $D$ such that

$$
F^{\Delta}(t)=f(t) \quad \text { for all } \quad t \in D .
$$

Definition 9 [6]. Suppose $f: \mathbb{T} \rightarrow \mathbb{R}$ is a regulated function. We say that the function $F$ as in Theorem 1 is a pre-antiderivative of $f$. The indefinite integral of a regulated function $f$ is defined by

$$
\int f(t) \Delta t=F(t)+C
$$

where $F$ is a pre-antiderivative of $f$ and $C$ is an arbitrary constant. The Cauchy integral is defined by

$$
\int_{s}^{t} f(t) \Delta t=F(t)-F(s) \text { for all } t, s \in \mathbb{T} .
$$

We say that a function $F: \mathbb{T} \rightarrow \mathbb{R}$ is an antiderivative of $f: \mathbb{T} \rightarrow \mathbb{R}$ if

$$
F^{\Delta}(t)=f(t) \quad \text { for all } \quad t \in \mathbb{T}^{k} \text {. }
$$


Theorem 2 [6]. Every rd-continuous function has an antiderivative.

Definition 10 [6]. For $p \in \mathcal{R}$, we define the generalized exponential function $e_{p}$ as the unique solution of the initial value problem

$$
x^{\Delta}(t)=p(t) x(t), \quad x(s)=1, \quad \text { where } \quad s \in \mathbb{T} .
$$

We give an explicit formula for $e_{p}(t, s)$ by

$$
e_{p}(t, s)=\exp \left(\int_{s}^{t} \xi_{\mu(v)}(p(v)) \Delta v\right) \quad \forall s, t \in \mathbb{T},
$$

where

$$
\xi_{\mu}(p)= \begin{cases}\frac{\log (1+\mu p)}{\mu} & \text { if } \mu \neq 0, \\ p & \text { if } \mu=0,\end{cases}
$$

with $\log$ is the principal logarithm function.

Lemma 1 [6]. For $p, q \in \mathcal{R}$, we define the functions $p \oplus q$ and $\ominus p$ by

$$
(p \oplus q)(t)=p(t)+q(t)+\mu(t) p(t) q(t) \quad \forall t \in \mathbb{T}^{k},
$$

and

$$
\ominus p(t)=-\frac{p(t)}{1+\mu(t) p(t)} \quad \forall t \in \mathbb{T}^{k}
$$

which are elements of $\mathcal{R}$.

Lemma 2 [6]. Let $p, q \in \mathcal{R}$. Then

(i) $e_{0}(t, s) \equiv 1$ and $e_{p}(t, t) \equiv 1$,

(ii) $e_{p}(\sigma(t), s)=(1+\mu(t) p(t)) e_{p}(t, s)$,

(iii) $\frac{1}{e_{p}(t, s)}=e_{\ominus p}(t, s)$,

(iv) $e_{p}(t, s)=\frac{1}{e_{p}(s, t)}=e_{\ominus p}(s, t)$,

(v) $e_{p}(t, s) e_{p}(s, r)=e_{p}(t, r)$,

(vi) $\left(\frac{1}{e_{p}(\cdot, s)}\right)^{\Delta}=-\frac{p(t)}{e_{p}^{\sigma}(\cdot, s)}$.

Lemma 3 [1]. If $p \in \mathcal{R}^{+}$, then

$$
0<e_{p}(t, s) \leq \exp \left(\int_{s}^{t} p(v) \Delta v\right) \quad \forall t \in \mathbb{T} .
$$

The following Schauder fixed point theorem plays important role to prove the existence results in the next section.

Theorem 3 [18, Schauder's fixed point theorem]. Suppose that $\Omega$ is a bounded closed convex nonempty subset of a Banach space $X$. Let $S: \Omega \rightarrow \Omega$ be a completely continuous mapping. Then $S$ has a fixed point in $\Omega$. 


\section{Positive periodic solutions}

Next theorem guarantee the existence of positive $\omega$-periodic solutions of (1.1).

Theorem 4. Assume that there exist positive constants $m$ and $M$, and a rd-continuous function $k \in C_{r d}\left(\left[t_{0}, \infty\right) \cap \mathbb{T}, \mathbb{R}\right)$ such that

$$
\begin{gathered}
a-k \in \mathcal{R}^{+}, \\
0<m \leq e_{\ominus(a-k)}\left(t, t_{0}\right) \leq M, \quad t \geq t_{0}, \\
\int_{t}^{t+\omega} \xi_{\mu(s)}[\ominus(a-k(s))] \Delta s=0, \quad t \geq t_{0},
\end{gathered}
$$

and

$$
\begin{gathered}
k(t) e_{\ominus(a-k)}\left(\sigma(t), t_{0}\right)=\int_{t_{0}}^{t}\left[r(s) \exp \left(\alpha \int_{t_{0}}^{s} \xi_{\mu(v)}[\ominus(a-k(v))] \Delta v\right)\right. \\
\left.-q(s) \exp \left(\beta \int_{t_{0}}^{s} \xi_{\mu(v)}[\ominus(a-k(v))] \Delta v\right)\right] \Delta s, \quad t \geq t_{0} .
\end{gathered}
$$

Then (1.1) has a positive $\omega$-periodic solution.

P r o o f. Let $X=B C_{r d}\left(\left[t_{0}, \infty\right) \cap \mathbb{T}, \mathbb{R}\right)$ be the Banach space of all bounded rd-continuous functions endowed with the supremum norm $\|x\|=\sup _{t \geq t_{0}}|x(t)|$. Consider the bounded closed convex nonempty subset $\Omega$ of $X$ as follows

$$
\begin{gathered}
\Omega=\left\{x \in X: x(t+\omega)=x(t), t \geq t_{0}, m \leq x(t) \leq M, t \geq t_{0},\right. \\
\left.\frac{1}{x^{\sigma}(t)} \int_{t_{0}}^{t}\left[r(s) x^{\alpha}(s)-q(s) x^{\beta}(s)\right] \Delta s=k(t), t \geq t_{0}\right\},
\end{gathered}
$$

and define the operator $S: \Omega \rightarrow X$ as follows

$$
(S x)(t)=\exp \left(\int_{t_{0}}^{t} \xi_{\mu(s)}\left[\ominus\left(a-\frac{1}{x^{\sigma}(s)} \int_{t_{0}}^{s}\left[r(v) x^{\alpha}(v)-q(v) x^{\beta}(v)\right] \Delta v\right)\right] \Delta s\right),
$$

for $t \geq t_{0}$. We will prove that $S \Omega \subset \Omega$. By using (3.1), for every $x \in \Omega$ and $t \geq t_{0}$ we obtain

$$
\begin{gathered}
(S x)(t)=\exp \left(\int_{t_{0}}^{t} \xi_{\mu(s)}\left[\ominus\left(a-\frac{1}{x^{\sigma}(s)} \int_{t_{0}}^{s}\left[r(v) x^{\alpha}(v)-q(v) x^{\beta}(v)\right] \Delta v\right)\right] \Delta s\right) \\
=e_{\ominus(a-k)}\left(t, t_{0}\right) \leq M .
\end{gathered}
$$

Also for $x \in \Omega$ and $t \geq t_{0}$ we have

$$
\begin{gathered}
(S x)(t)=\exp \left(\int_{t_{0}}^{t} \xi_{\mu(s)}[\ominus(\right. \\
\left.\left.\left(a-\frac{1}{x^{\sigma}(s)} \int_{t_{0}}^{s}\left[r(v) x^{\alpha}(v)-q(v) x^{\beta}(v)\right] \Delta v\right)\right] \Delta s\right) \\
=e_{\ominus(a-k)}\left(t, t_{0}\right) \geq m .
\end{gathered}
$$


From (3.3), for every $x \in \Omega$ and $t \geq t_{0}$ we obtain

$$
\begin{gathered}
k(t)(S x)^{\sigma}(t)=k(t) \exp \left(\int_{t_{0}}^{\sigma(t)} \xi_{\mu(s)}\left[\ominus\left(a-\frac{1}{x^{\sigma}(s)} \int_{t_{0}}^{s}\left[r(v) x^{\alpha}(v)-q(v) x^{\beta}(v)\right] \Delta v\right)\right] \Delta s\right) \\
=k(t) \exp \left(\int_{t_{0}}^{\sigma(t)} \xi_{\mu(s)}[\ominus(a-k(s))] \Delta s\right)=k(t) e_{\ominus(a-k)}\left(\sigma(t), t_{0}\right) \\
=\int_{t_{0}}^{t}\left[r(s) \exp \left(\alpha \int_{t_{0}}^{s} \xi_{\mu(v)}[\ominus(a-k(v))] \Delta v\right)-q(s) \exp \left(\beta \int_{t_{0}}^{s} \xi_{\mu(v)}[\ominus(a-k(v))] \Delta v\right)\right] \Delta s \\
=\int_{t_{0}}^{t}\left[r(s)(S x)^{\alpha}(s)-q(s)(S x)^{\beta}(s)\right] \Delta s .
\end{gathered}
$$

Finally we will prove that for $x \in \Omega, t \geq t_{0}$ the function $S x$ is $\omega$-periodic. By using (3.2), for $x \in \Omega$ and $t \geq t_{0}$ we get

$$
\begin{gathered}
(S x)(t+\omega)=\exp \left(\int_{t_{0}}^{t+\omega} \xi_{\mu(s)}\left[\ominus\left(a-\frac{1}{x^{\sigma}(s)} \int_{t_{0}}^{s}\left[r(v) x^{\alpha}(v)-q(v) x^{\beta}(v)\right] \Delta v\right)\right] \Delta s\right) \\
=\exp \left(\int_{t_{0}}^{t} \xi_{\mu(s)}\left[\ominus\left(a-\frac{1}{x^{\sigma}(s)} \int_{t_{0}}^{s}\left[r(v) x^{\alpha}(v)-q(v) x^{\beta}(v)\right] \Delta v\right)\right] \Delta s\right) \\
\times \exp \left(\int_{t}^{t+\omega} \xi_{\mu(s)}\left[\ominus\left(a-\frac{1}{x^{\sigma}(s)} \int_{t_{0}}^{s}\left[r(v) x^{\alpha}(v)-q(v) x^{\beta}(v)\right] \Delta v\right)\right] \Delta s\right) \\
=(S x)(t) \exp \left(\int_{t}^{t+\omega} \xi_{\mu(s)}[\ominus(a-k(s))] \Delta s\right)=(S x)(t) .
\end{gathered}
$$

So $S x$ is $\omega$-periodic on $\left[t_{0}, \infty\right) \cap \mathbb{T}$. Hence, $S \Omega \subset \Omega$.

Now, we need to prove that the mapping $S$ is completely continuous. So we will show that the mapping $S$ is continuous. Let $x_{i} \in \Omega$ be such that $x_{i} \longrightarrow x \in \Omega$ as $i \longrightarrow \infty$. For $t \geq t_{0}$, we have

$$
\begin{aligned}
\mid\left(S x_{i}\right)(t)- & (S x)(t)|=| \exp \left(\int_{t_{0}}^{t} \xi_{\mu(s)}\left[\ominus\left(a-\frac{1}{x_{i}^{\sigma}(s)} \int_{t_{0}}^{s}\left[r(v) x_{i}^{\alpha}(v)-q(v) x_{i}^{\beta}(v)\right] \Delta v\right)\right] \Delta s\right) \\
& -\exp \left(\int_{t_{0}}^{t} \xi_{\mu(s)}\left[\ominus\left(a-\frac{1}{x^{\sigma}(s)} \int_{t_{0}}^{s}\left[r(v) x^{\alpha}(v)-q(v) x^{\beta}(v)\right] \Delta v\right)\right] \Delta s\right) \mid .
\end{aligned}
$$

By applying the Lebesgue dominated convergence theorem we obtain that

$$
\lim _{i \longrightarrow \infty}\left\|S x_{i}-S x\right\|=0 .
$$

Therefore $S$ is continuous.

Next, we are going to prove that $S \Omega$ is relatively compact by applying the Arzela-Ascoli theorem. The uniform boundedness of $S \Omega$ follows from the definition of $\Omega$. For $t \geq t_{0}$ and $x \in \Omega$ we have

$$
\begin{gathered}
\left|(S x)^{\Delta}(t)\right|=\left|-\left(a-\frac{1}{x^{\sigma}(t)} \int_{t_{0}}^{t}\left[r(v) x^{\alpha}(v)-q(v) x^{\beta}(v)\right] \Delta v\right)\right| \\
\times \exp \left(\int_{t_{0}}^{\sigma(t)} \xi_{\mu(s)}\left[\ominus\left(a-\frac{1}{x^{\sigma}(s)} \int_{t_{0}}^{s}\left[r(v) x^{\alpha}(v)-q(v) x^{\beta}(v)\right] \Delta v\right)\right] \Delta s\right) \\
=\left|\ominus\left(a-\frac{1}{x^{\sigma}(t)} \int_{t_{0}}^{t}\left[r(v) x^{\alpha}(v)-q(v) x^{\beta}(v)\right] \Delta v\right)\right| \\
\times \exp \left(\int_{t_{0}}^{t} \xi_{\mu(s)}\left[\ominus\left(a-\frac{1}{x^{\sigma}(s)} \int_{t_{0}}^{s}\left[r(v) x^{\alpha}(v)-q(v) x^{\beta}(v)\right] \Delta v\right)\right] \Delta s\right) \\
=|\ominus(a-k(t))| e_{\ominus}(a-k) \\
\end{gathered}
$$


which implies that the family $S \Omega$ is equicontinuous. By using the Arzela-Ascoli theorem $S \Omega$ is relatively compact. Therefore, $S$ is completely continuous. By Theorem 3 there is an $x_{0} \in \Omega$ such that $S x_{0}=x_{0}$. We see that $x_{0}$ is a positive $\omega$-periodic solution of (1.1). The proof is complete.

To illustrate the applications of Theorem 4 we give the following example.

Example 2. Consider the dynamic equation on $\mathbb{T}=\pi \mathbb{Z}$ then $\mu(t)=\pi$,

$$
x^{\triangle \Delta}(t)+a\left(x^{\sigma}\right)^{\Delta}(t)+q(t) x^{\beta}(t)-r(t) x^{\alpha}(t)=0, \quad t \geq t_{0} .
$$

We take $t_{0} \in \mathbb{T}$ which

$$
a=\frac{e^{\cos t_{0}}-1}{\pi} \geq 0, \quad k(t)=a-\frac{e^{\cos t}-1}{\pi}, \quad \omega=4 \pi, \quad \alpha, \beta \in(0, \infty) .
$$

Then for the conditions (3.1), (3.2) and $\omega=4 \pi$ we obtain

$$
\begin{gathered}
1+\mu(t)(a-k(t))=e^{\cos t}>0, \quad t \geq t_{0}, \quad \text { then } \quad a-k \in \mathcal{R}^{+}, \\
\int_{t}^{t+\omega} \xi_{\mu(s)}[\ominus(a-k(s))] \Delta s=\int_{t}^{t+4 \pi} \frac{1}{\mu(s)} \log [1+\mu(s)(\ominus(a-k(s)))] \Delta s \\
=\int_{t}^{t+4 \pi} \frac{1}{\pi} \log \left[\frac{-(a-k(s))}{1+\mu(s)(a-k(s))} \mu(s)+1\right] \Delta s=-\int_{t}^{t+4 \pi} \frac{1}{\pi} \log [1+\mu(t)(a-k(s))] \Delta s \\
=-\int_{t}^{t+4 \pi} \frac{1}{\pi} \cos (s) \Delta s=\left.\frac{1}{2} \cos (s)\right|_{t} ^{t+4 \pi}=0, \quad t \geq t_{0},
\end{gathered}
$$

and

$$
\begin{gathered}
e_{\ominus(a-k)}\left(t, t_{0}\right)=\exp \int_{t_{0}}^{t} \xi_{\mu(s)}[\ominus(a-k(s))] \Delta \\
=\exp \int_{t_{0}}^{t} \frac{1}{\mu(s)} \log [1+\mu(s)(\ominus(a-k(s)))] \Delta s=e^{\left(\cos t-\cos t_{0}\right) / 2}, \quad t \geq t_{0} .
\end{gathered}
$$

We take $m=e^{-1}$ and $M=e$, then

$$
0<m \leq e_{\ominus(a-k)}\left(t, t_{0}\right) \leq M, \quad t \geq t_{0} .
$$

Also, we put

$$
r(t)=\frac{e^{\frac{1}{2} \cos t}-e^{-\frac{1}{2} \cos t}}{\pi} e^{-\frac{\alpha}{2}\left(\cos t-\cos t_{0}\right)}\left(a+\frac{1}{\pi}\right) e^{-\frac{1}{2} \cos t_{0}},
$$

and

$$
q(t)=\frac{e^{-\frac{1}{2} \cos t}-e^{\frac{1}{2} \cos t}}{\pi^{2}} e^{-\frac{\beta}{2}\left(\cos t-\cos t_{0}\right)} e^{-\frac{1}{2} \cos t_{0}}
$$

then

$$
\begin{gathered}
k(t) e_{\ominus(a-k)}\left(\sigma(t), t_{0}\right)=\left(a-\frac{e^{\cos t}-1}{\pi}\right) \exp \int_{t_{0}}^{\sigma(t)} \xi_{\mu(s)}[\ominus(a-k(s))] \Delta s \\
=\left(a+\frac{1}{\pi}\right) e^{-\frac{1}{2}\left(\cos (t)+\cos t_{0}\right)}-\frac{1}{\pi} e^{\frac{1}{2}\left(\cos (t)-\cos t_{0}\right)}, \quad t \geq t_{0},
\end{gathered}
$$


and

$$
\begin{gathered}
\int_{t_{0}}^{t} r(s) \exp \left(\alpha \int_{t_{0}}^{s} \xi_{\mu(v)}[\ominus(a-k(v))] \Delta v\right)-q(s) \exp \left(\beta \int_{t_{0}}^{s} \xi_{\mu(v)}[\ominus(a-k(v))] \Delta v\right) \Delta s \\
=\int_{t_{0}}^{t}\left[\frac{e^{\frac{1}{2} \cos s}-e^{-\frac{1}{2} \cos s}}{\pi}\left(a+\frac{1}{\pi}\right) e^{-\frac{1}{2} \cos t_{0}}-\frac{e^{-\frac{1}{2} \cos s}-e^{\frac{1}{2} \cos s}}{\pi^{2}} e^{-\frac{1}{2} \cos t_{0}}\right] \Delta s \\
=\left(a+\frac{1}{\pi}\right) e^{-\frac{1}{2}\left(\cos t+\cos t_{0}\right)}-\frac{1}{\pi} e^{\frac{1}{2}\left(\cos t-\cos t_{0}\right)}, \quad t \geq t_{0} .
\end{gathered}
$$

All conditions of Theorem 4 are satisfied. Thus (3.4) has a positive $\omega=4 \pi$-periodic solution

$$
x(t)=e_{\ominus(a-k)}\left(t, t_{0}\right)=e^{\frac{1}{2}\left(\cos t-\cos t_{0}\right)}, \quad t \geq t_{0},
$$

with $x\left(t_{0}\right)=1$ and

$$
x^{\Delta}\left(t_{0}\right)+a x^{\sigma}\left(t_{0}\right)=e^{-\frac{1}{2} \cos t_{0}}\left(\frac{e^{-\frac{1}{2} \cos t_{0}}-e^{\frac{1}{2} \cos t_{0}}}{\pi}\right)+a e^{-\cos t_{0}}=0 .
$$

\section{Exponential stability of positive periodic solutions}

In this section, we will prove the exponential stability of a positive $\omega$-periodic solution of (1.1). Let $x_{1}$ be the positive $\omega$-periodic solution of (1.1) with the initial condition $x_{1}\left(t_{0}\right)=1$ and $x_{1}^{\Delta}\left(t_{0}\right)+a x_{1}^{\sigma}\left(t_{0}\right)=0$. Let $x$ be the another positive $\omega$-periodic solution of (1.1) with the initial condition $x\left(t_{0}\right)=c_{1}>0, c_{1} \neq 1$ and $x^{\Delta}\left(t_{0}\right)+a x^{\sigma}\left(t_{0}\right)=0$. Let

$$
y(t)=x(t)-x_{1}(t), t \geq t_{0} .
$$

After integration of (1.1), we obtain

$$
\int_{t_{0}}^{t} x^{\Delta \Delta}(s) \Delta s+a \int_{t_{0}}^{t}\left(x^{\sigma}\right)^{\Delta}(s) \Delta s+\int_{t_{0}}^{t}\left[q(s) x^{\beta}(s)-r(s) x^{\alpha}(s)\right] \Delta s=0,
$$

so

$$
x^{\Delta}(t)-x^{\Delta}\left(t_{0}\right)+a x^{\sigma}(t)-a x^{\sigma}\left(t_{0}\right)=\int_{t_{0}}^{t}\left[r(s) x^{\alpha}(s)-q(s) x^{\beta}(s)\right] \Delta s .
$$

Then

$$
x^{\Delta}(t)+a x^{\sigma}(t)=\int_{t_{0}}^{t}\left[r(s) x^{\alpha}(s)-q(s) x^{\beta}(s)\right] \Delta s .
$$

In a similar way one can easily show that

$$
x_{1}^{\Delta}(t)+a x_{1}^{\sigma}(t)=\int_{t_{0}}^{t}\left[r(s) x_{1}^{\alpha}(s)-q(s) x_{1}^{\beta}(s)\right] \Delta s .
$$

Therefore

$$
\begin{gathered}
y^{\Delta}(t)=x^{\Delta}(t)-x_{1}^{\Delta}(t)=-a\left[x^{\sigma}(t)-x_{1}^{\sigma}(t)\right] \\
+\int_{t_{0}}^{t}\left[r(s)\left(x^{\alpha}(s)-x_{1}^{\alpha}(s)\right)-q(s)\left(x^{\beta}(s)-x_{1}^{\beta}(s)\right)\right] \Delta s .
\end{gathered}
$$

This implies

$$
y^{\Delta}(t)=-a y^{\sigma}(t)+\int_{t_{0}}^{t}\left[r(s)\left(x^{\alpha}(s)-x_{1}^{\alpha}(s)\right)-q(s)\left(x^{\beta}(s)-x_{1}^{\beta}(s)\right)\right] \Delta s .
$$


By using the mean value theorem, we get

$$
\begin{gathered}
y^{\Delta}(t)=-a y^{\sigma}(t)+\int_{t_{0}}^{t}\left[r(s) \alpha x_{*}^{\alpha-1}(s)\left(x(s)-x_{1}(s)\right)-q(s) \beta x_{0}^{\beta-1}(s)\left(x(s)-x_{1}(s)\right)\right] \Delta s \\
=-a y^{\sigma}(t)+\int_{t_{0}}^{t}\left[r(s) \alpha x_{*}^{\alpha-1}(s)-q(s) \beta x_{0}^{\beta-1}(s)\right] y(s) \Delta s, \quad t \geq t_{0},
\end{gathered}
$$

for $x_{*}, x_{0} \in\left[x, x_{1}\right]$ or $x_{*}, x_{0} \in\left[x_{1}, x\right]$.

For $m \leq x(t) \leq M$, we suppose that the function

$$
f(t, x(t))=-a x^{\sigma}(t)+\int_{t_{0}}^{t}\left[r(s) x^{\alpha}(s)-q(s) x^{\beta}(s)\right] \Delta s, \quad t \geq t_{0},
$$

is Lipschitzian in second argument.

Definition 11. Assume that $x_{1}$ is the positive w-periodic solution of (1.1). If there exist positive constants $K_{x_{1}}$ and $\lambda$ for every positive $\omega$-periodic solution $x$ of (1.1) such that

$$
0<m_{*} \leq x(t) \leq M_{*}, \quad m_{*} \leq m, \quad M_{*} \geq M, \quad x^{\Delta}\left(t_{0}\right)+a x^{\sigma}\left(t_{0}\right)-x_{1}^{\Delta}\left(t_{0}\right)-a x_{1}^{\sigma}\left(t_{0}\right)=0
$$

and

$$
\left|x(t)-x_{1}(t)\right|<K_{x_{1}} e_{\odot \lambda}(t, 0) \quad \forall t>t_{0},
$$

then $x_{1}$ is said to be exponentially stable.

In the next theorem, we prove the exponential stability of the positive periodic solution $x_{1}$ of (1.1).

Theorem 5. Assume that $q, r \in C_{r d}\left(\left[t_{0}, \infty\right) \cap \mathbb{T},\left(t_{0}, \infty\right)\right)$ and there exist positive constants $m$ and $M$, and a function $k \in C_{r d}\left(\left[t_{0}, \infty\right) \cap \mathbb{T}, \mathbb{R}\right)$ such that $(3.1)-(3.3)$ hold. Let $a>0,0<\alpha<\beta<1$ and there exist constants $m_{*}, M_{*} \in(0, \infty)$ such that $m_{*} \leq m, M_{*} \geq M$ and

$$
\alpha m_{*}^{\alpha-1} r(t)-\beta M_{*}^{\beta-1} q(t) \leq 0 \text { for } t \geq t_{0} .
$$

Then (1.1) has a positive $\omega$-periodic solution which is exponentially stable.

P r o o f. Conditions (3.1)-(3.3) imply that (1.1) has a positive $\omega$-periodic solution $x_{1}$. Let $x$ be a positive $\omega$-periodic solution of (1.1) such that $m_{*} \leq x(t) \leq M_{*}$,

$$
x^{\Delta}\left(t_{0}\right)+a x^{\sigma}\left(t_{0}\right)-x_{1}^{\Delta}\left(t_{0}\right)-a x_{1}^{\sigma}\left(t_{0}\right)=0 .
$$

We prove that there exists $\lambda \in(0, \infty)$ such that

$$
\left|x(t)-x_{1}(t)\right|<K_{x_{1}} e_{\ominus \lambda}(t, 0), \quad t \geq t_{0},
$$

where $K_{x_{1}}=e_{\lambda}\left(t_{0}, 0\right)\left|y\left(t_{0}\right)\right|+1$.

We define the Lyapunov function

$$
L(t)=|y(t)| e_{\lambda}(t, 0), \quad t \geq t_{0}, \quad \lambda \in(0, a) .
$$

For $t>t_{0}$, we assume that $L(t)<K_{x_{1}}$. On the other hand there exists $t_{*} \geq t_{0}$ such that $L\left(t_{*}\right)=K_{x_{1}}$ and $L(t)<K_{x_{1}}$ for $t \in\left[t_{0}, t_{*}\right)$. By calculation of the upper left delta derivative of $L(t)$ along the solution of (4.1), we get

$$
\begin{gathered}
(L(t))^{\Delta^{-} \leq-a\left|y^{\sigma}(t)\right| e_{\lambda}(t, 0)}+e_{\lambda}(t, 0) \int_{t_{0}}^{t}\left[r(s) \alpha x_{*}^{\alpha-1}(s)-q(s) \beta x_{0}^{\beta-1}(s)\right]|y(s)| \Delta s \\
+\lambda\left|y^{\sigma}(t)\right| e_{\lambda}(t, 0), \quad t \geq t_{0} .
\end{gathered}
$$


For $t=t_{*}$ we have

$$
\begin{gathered}
0 \leq\left(L\left(t_{*}\right)\right)^{\Delta^{-}} \leq(\lambda-a)\left|y^{\sigma}\left(t_{*}\right)\right| e_{\lambda}\left(t_{*}, 0\right)+e_{\lambda}\left(t_{*}, 0\right) \int_{t_{0}}^{t_{*}}\left[r(s) \alpha x_{*}^{\alpha-1}(s)-q(s) \beta x_{0}^{\beta-1}(s)\right]|y(s)| \Delta s \\
\leq(\lambda-a)\left|y^{\sigma}\left(t_{*}\right)\right| e_{\lambda}\left(t_{*}, 0\right)+e_{\lambda}\left(t_{*}, 0\right) \int_{t_{0}}^{t_{*}}\left[\alpha m_{*}^{\alpha-1} r(s)-\beta M_{*}^{\beta-1} q(s)\right]|y(s)| \Delta s \\
\leq(\lambda-a)\left|y^{\sigma}\left(t_{*}\right)\right| e_{\lambda}\left(t_{*}, 0\right) .
\end{gathered}
$$

If $y(t)>0, t \geq t_{0}$, then from (4.1) it follows that, for $t \geq t_{0}$, the function $y$ is decreasing. If $y(t)<0, t \geq t_{0}$, then $y$ is increasing for $t \geq t_{0}$. We conclude that $|y(t)|, t \geq t_{0}$ has decreasing character. Then we obtain

$$
0 \leq\left(L\left(t_{*}\right)\right)^{\Delta^{-}} \leq(\lambda-a)\left|y\left(t_{*}\right)\right| e_{\lambda}\left(t_{*}, 0\right) \leq(\lambda-a) K_{x_{1}}<0,
$$

which is a contradiction. Hence, we get

$$
|y(t)| e_{\lambda}(t, 0)<K_{x_{1}} \quad \text { for } \quad t \geq t_{0} \quad \text { and some } \quad \lambda \in(0, a) .
$$

The proof is complete.

\section{Application in a pipe-tank configuration}

In [11], Cid et al. reformulated the problem of fluid motion in the pipe into the following periodic boundary value problem

$$
\left\{\begin{array}{l}
u^{\prime \prime}(t)+a u^{\prime}(t)=\frac{1}{u(t)}\left(e(t)-b\left(u^{\prime}(t)^{2}\right)-c, \quad t \in[0, \omega],\right. \\
u(0)=u(\omega), \quad u^{\prime}(0)=u^{\prime}(\omega)
\end{array}\right.
$$

where $a \geq 0, b>1, c>0$ and $e$ is $\omega$-periodic continuous on $\mathbb{R}$. By using the change of variables $u=x^{1 /(b+1)}$, the singular problem (5.1) can be transformed to the following regular problem

$$
\left\{\begin{array}{l}
x^{\prime \prime}(t)+a x^{\prime}(t)+q(t) x^{\beta}(t)-r(t) x^{\alpha}(t)=0, \quad t \in[0, \omega], \\
x(0)=x(\omega), \quad x^{\prime}(0)=x^{\prime}(\omega),
\end{array}\right.
$$

where

$$
r(t)=(b+1) e(t), \quad q(t)=(b+1) c, \quad \alpha=\frac{b-1}{b+1}, \quad \beta=\frac{b}{b+1},
$$

with $0<\alpha<\beta<1$.

We will give new sufficient conditions ensuring the existence and the exponential stability of positive $\omega$-periodic solutions of the following dynamic equation

$$
x^{\triangle \Delta}(t)+a\left(x^{\sigma}\right)^{\Delta}(t)+(b+1)\left[c x^{\beta}(t)-e(t) x^{\alpha}(t)\right]=0, \quad t \geq t_{0} .
$$

With respect to Theorems 4 and 5 , we obtain the following theorem.

Theorem 6. Assume that $a>0,0<\alpha<\beta<1$ and there exist positive constants $m$ and $M$, and a function $k \in C_{r d}\left(\left[t_{0}, \infty\right) \cap \mathbb{T}, \mathbb{R}\right)$ such that (3.1) and (3.2) hold and

$$
\begin{gathered}
k(t) e_{\ominus(a-k)}\left(\sigma(t), t_{0}\right)=(b+1) \int_{t_{0}}^{t} e(s) \exp \left(\alpha \int_{t_{0}}^{s} \xi_{\mu(v)}[\ominus(a-k(v))] \Delta v\right) \\
-c \exp \left(\beta \int_{t_{0}}^{s} \xi_{\mu(v)}[\ominus(a-k(v))] \Delta v\right) \Delta s, \quad t \geq t_{0} .
\end{gathered}
$$

Then (5.2) has a positive $\omega$-periodic solution. 
Theorem 7. Assume that $e \in C_{r d}\left(\left[t_{0}, \infty\right) \cap \mathbb{T},\left(t_{0}, \infty\right)\right), a>0,0<\alpha<\beta<1, c>0$ and there exist positive constants $m$ and $M$, and a function $k \in C_{r d}\left(\left[t_{0}, \infty\right) \cap \mathbb{T}, \mathbb{R}\right)$ such that (3.1), (3.2) and (5.3) hold. Let, in addition, there exist constants $m_{*}, M_{*} \in(0, \infty)$ such that $m_{*} \leq m, M_{*} \geq M$ and

$$
\alpha m_{*}^{\alpha-1} e(t)-\beta M_{*}^{\beta-1} c \leq 0 \text { for } t \geq t_{0} .
$$

Then (5.2) has a positive $\omega$-periodic solution which is exponentially stable.

\section{Conclusion}

In this paper, we provided the existence and exponential stability of positive periodic solutions with sufficient conditions for second-order dynamic equations on time scales. The main tools of this paper are the fixed point method and the Lyapunov method. However, by introducing new fixed mappings and suitable Lyapunov functionals, we get new existence and exponential stability conditions. An example illustrating our results is presented. The obtained results have a contribution to the related literature, and they improve and extend the results in [13] from the case of second-order differential equations to that case with second-order dynamic equations on time scales. It seems that the results of this paper can be extended to cover the case of delay second-order dynamic equations.

\section{Acknowledgements}

The authors would like to thank the anonymous referee for his/her valuable comments and good advice.

\section{REFERENCES}

1. Adivar M., Raffoul Y.N. Existence of periodic solutions in totally nonlinear delay dynamic equations. Electron. J. Qual. Theory Differ. Equ., 2009. Vol. 2009, No. 1. P. 1-20. DOI: 10.14232/ejqtde.2009.4.1

2. Ardjouni A., Djoudi A. Existence of positive periodic solutions for nonlinear neutral dynamic equations with variable delay on a time scale. Malaya Journal of Matematik, 2013. Vol. 2, No. 1. P. 60-67.

3. Ardjouni A., Djoudi A. Existence of periodic solutions for nonlinear neutral dynamic equations with functional delay on a time scale. Acta Univ. Palack. Olomuc. Fac. Rerum Natur. Math., 2013. Vol. 52, No. 1. P. $5-19$.

4. Ardjouni A., Djoudi A. Existence of periodic solutions for nonlinear neutral dynamic equations with variable delay on a time scale. Commun. Nonlinear Sci. Numer. Simul., 2012. Vol. 17. P. 3061-3069. DOI: $10.1016 / \mathrm{j} . \mathrm{cns} n s .2011 .11 .026$

5. Ardjouni A., Djoudi A. Periodic solutions in totally nonlinear dynamic equations with functional delay on a time scale. Rend. Sem. Mat. Univ. Politec. Torino, 2010. Vol. 68, No. 4. P. 349-359.

6. Bohner M., Peterson A. Dynamic Equations on Time Scales, An Introduction with Applications. Birkhäuser, Boston, 2001. 358 p. DOI: 10.1007/978-1-4612-0201-1

7. Bohner M., Peterson A. Advances in Dynamic Equations on Time Scales. Birkhäuser, Boston, 2003. 348 p. DOI: $10.1007 / 978-0-8176-8230-9$

8. Bouchelaghem F., Ardjouni A., Djoudi A. Existence and stability of positive periodic solutions for delay nonlinear dynamic equations. Nonlinear Stud., 2018. Vol. 25, No. 1. P. 191-202.

9. Bouchelaghem F., Ardjouni A., Djoudi A. Existence of positive solutions of delay dynamic equations. Positivity, 2017. Vol. 21, No. 4. P. 1483-1493. DOI: 10.1007/s11117-017-0480-2

10. Bouchelaghem F., Ardjouni A., Djoudi A. Existence of positive periodic solutions for delay dynamic equations. Proyecciones (Antofagasta), 2017. Vol. 36, No. 3. P. 449-460. DOI: $10.4067 / \mathrm{S} 0716-09172017000300449$

11. Cid J. Á., Propst G., Tvrdý M. On the pumping effect in a pipe/tank flow configuration with friction. Phys. D: Nonlinear Phenomena, 2014. Vol. 273/274. P. 28-33. DOI: 10.1016/j.physd.2014.01.010 
12. Culáková I., Hanuštiaková L'., Olach R. Existence for positive solutions of second-order neutral nonlinear differential equations. Appl. Math. Lett., 2009. Vol. 22, No. 7. P. 1007-1010. DOI: $10.1016 /$ j.aml.2009.01.009

13. Dorociaková B., Michalková M., Olach R., Sága M. Existence and Stability of Periodic Solution Related to Valveless Pumping. Math. Probl. Eng., 2018. Vol. 2018. Art. ID 3982432. P. 1-8. DOI: $10.1155 / 2018 / 3982432$

14. Gouasmia M., Ardjouni A., Djoudi A. Periodic and nonnegative periodic solutions of nonlinear neutral dynamic equations on a time scale. Int. J. Anal. Appl., 2018. Vol. 16, No. 2. P. 162-177. DOI: $10.28924 / 2291-8639-16-2018-162$

15. Hilger S. Ein Masskettenkalkul mit Anwendung auf Zentrumsmanningfaltigkeiten. PhD thesis, Universitat Wurzburg, 1988.

16. Kaufmann E. R., Raffoul Y. N. Periodic solutions for a neutral nonlinear dynamical equation on a time scale. J. Math. Anal. Appl., 2006. Vol. 319. P. 315-325. DOI: 10.1016/j.jmaa.2006.01.063

17. Lakshmikantham V., Sivasundaram S., Kaymarkcalan B. Dynamic Systems on Measure Chains. Dordrecht: Kluwer Academic Publishers, 1996. 294 p. DOI: 10.1007/978-1-4757-2449-3

18. Smart D. R. Fixed Points Theorems. Cambridge, UK: Cambridge Univ. Press, 1980. 104 p. 